\title{
A Multi-State Survey of Consumer Food-Handling and Food-Consumption Practices
}

\author{
Sean F. Altekruse, DVM, Samantha Yang, MPH, Babagaleh B. Timbo, DrPH, Frederick J. Angulo, DVM
}

\begin{abstract}
Introduction: In the United States, foodborne infections cause an estimated 6.5-33 million illnesses a year. Also included in the burden of foodborne illnesses are sequelae such as hemolytic uremic syndrome, Guillain-Barré syndrome, and reactive arthritis. Surveillance for risky food-handling and food-consumption practices can be used to identify high-risk populations, develop educational efforts, and evaluate progress toward risk reduction.
\end{abstract}

Design: $\quad$ In 1995 and 1996, Behavioral Risk Factor Surveillance System interviews of 19,356 adults in eight states (1995: Colorado, Florida, Missouri, New York, and Tennessee; 1996: Indiana, New Jersey, and South Dakota) included questions related to food-handling and/or food-consumption practices. Risky food-handling and food-consumption practices were not uncommon. Overall, $19 \%$ of respondents did not adequately wash hands or cutting boards after contact with raw meat or chicken. During the previous year, $20 \%$ ate pink hamburgers, $50 \%$ ate undercooked eggs, $8 \%$ ate raw oysters, and $1 \%$ drank raw milk. Men were more likely to report risky practices than women. The prevalence of most risky behaviors increased with increasing socioeconomic status.

Conclusion: Targeted education efforts may reduce the frequency of these behaviors. Periodic surveillance can be used to assess effectiveness. In addition to consumer education, prevention efforts are needed throughout the food chain including on the farm, in processing, distribution, and at retail.

Medical Subject Headings (MeSH): food poisoning, behavior, demography (Am J Prev Med 1999;16(3):216-221) (c) 1999 American Journal of Preventive Medicine

\section{Introduction}

$\mathrm{E}$ ach year, foodborne diseases affect an estimated 6.5-33 million people in the United States, ${ }^{1}$ with medical costs and productivity losses estimated at $\$ 9.3$ to $\$ 12.9$ billion dollars. ${ }^{1}$ This burden of foodborne illnesses includes several chronic sequelae such as hemolytic uremic syndrome, Guillain-Barré syndrome, and reactive arthritis. ${ }^{2}$ Changes in population demographics, the foods we eat, and where they come from have facilitated the emergence of new pathogens and food vehicles for their transmission, including Salmonella serotype enteritidis in eggs, Escherichia coli O157:H7 in ground beef and fresh produce, and Campylobacter jejuni in raw poultry. ${ }^{2}$ Foodborne disease prevention technologies include milk pasteurization, sanitary controls on the farm, and Hazard Analysis and

From the Food and Drug Administration, Center for Food Safety and Applied Nutrition, Office of Scientific Assessment and Support, Division of Market Studies, Epidemiology Branch (HFS-728) (Altekruse, Timbo), Washington, DC; and Centers for Disease Control and Prevention, National Center for Infectious Diseases, Division of Bacterial and Mycotic Diseases, Foodborne and Diarrheal Diseases Branch (Yang, Angulo), Atlanta, GA.

Address correspondence to: Samantha Yang, MPH, CDC, NCID, FDDB (A38), 1600 Clifton Road, Atlanta, GA 30333.
Critical Control Point (HACCP) programs, industry efforts to identify and control hazards in the food chain. ${ }^{3}$ The National Food Safety Initiative (FSI), which began in 1997, has focused attention on improving data on how pathogens, foods, and people interact; developing coordinated regulatory responses; and establishing a long-term strategy to address hazards in the food chain. ${ }^{3}$ Development of consumer educational efforts to promote safe food-handling and food-consumption practices is an FSI priority. ${ }^{3,4}$ Behavioral surveillance is used to develop targeted educational efforts and evaluate progress toward risk reduction. ${ }^{5-9}$ In this study, we analyzed Behavioral Risk Factor Surveillance System (BRFSS) data on risky food-handling and food-consumption behaviors from interviews conducted in eight states during 1995 and 1996.

\section{Methods}

BRFSS is a state-based telephone survey of adults that uses random-digit dialing on the basis of either the Waksberg or disproportionate stratified random sample method. ${ }^{10}$ In 1995, Colorado, Florida, Missouri, New York, and Tennessee added to their BRFSS surveys questions about food-handling and food-consumption 
practices. Respondents were asked about practices after contact with raw meat or chicken, regarding (1) handwashing, and (2) cutting boards. Specifically, they were asked, "Do you:" and were read four responses: "Continue cooking," "Rinse and/or wipe (hands or cutting board, respectively)," "Wash (hands with soap and water or cutting boards with soap or bleach and water)" then continue cooking, and "You don't handle uncooked meat or poultry." Practices defined as safe were washing hands with soap and water and washing cutting boards with soap or bleach and water before continuing to cook. Respondents were also asked how often in the previous year they ate hamburgers that were pink at the center; undercooked eggs, and/or raw oysters, and whether they drank unpasteurized milk. In 1996, Indiana and New Jersey added these questions to their BRFSS surveys and South Dakota added only questions regarding consumption of undercooked hamburgers and undercooked eggs to their BRFSS survey. Participants who responded "don't know," "not sure," gave unlisted responses, or refused to answer questions were excluded from relevant analyses. The respondents' counties of residence were classified as rural $(<20,000$ population), suburban $(>20,000$ population but not urban), or urban (central counties in metropolitan areas with populations of $\geq 1$ million). ${ }^{11}$ Salary was self-reported annual household income. Prevalences and frequencies of risky behaviors were calculated using Statistical Analysis System (SAS). ${ }^{12}$ SUrvey DAta ANalysis (SUDAAN) was used to determine weighted prevalences ${ }^{13}$ on the basis of respondent's probability of selection and age-, race-, and gender-specific distributions of the 1990 census population.

\section{Results}

A total of 19,356 adults were interviewed: 2,461 in Colorado; 3,335 in Florida, 2,212 in Indiana; 1,572 in Missouri; 3,149 in New Jersey; 2,477 in New York; 2,110 in South Dakota; and 2,040 in Tennessee. Overall, 55\% of respondents were $30-59$ years of age, $59 \%$ were women, $80 \%$ were white, $52 \%$ had attended at least some college, $48 \%$ reported household incomes $<\$ 35,000$, and $76 \%$ resided in urban areas. The Council of the American Survey Research Organization response rate was $64 \% .{ }^{14}$

In this survey, $19 \%$ of respondents reported not routinely washing their hands with soap after handling raw meat or chicken. The prevalence of this risky behavior varied from $15 \%$ in Tennessee to $23 \%$ in Colorado (Table 1), was significantly higher for men $(25 \%)$ than women $(14 \%)$, and declined with increasing age (Table 2). In addition, the prevalence was significantly higher for whites (19\%) than blacks (13\%), and increased with increasing education and increasing income.

Overall, $19 \%$ of respondents reported not routinely

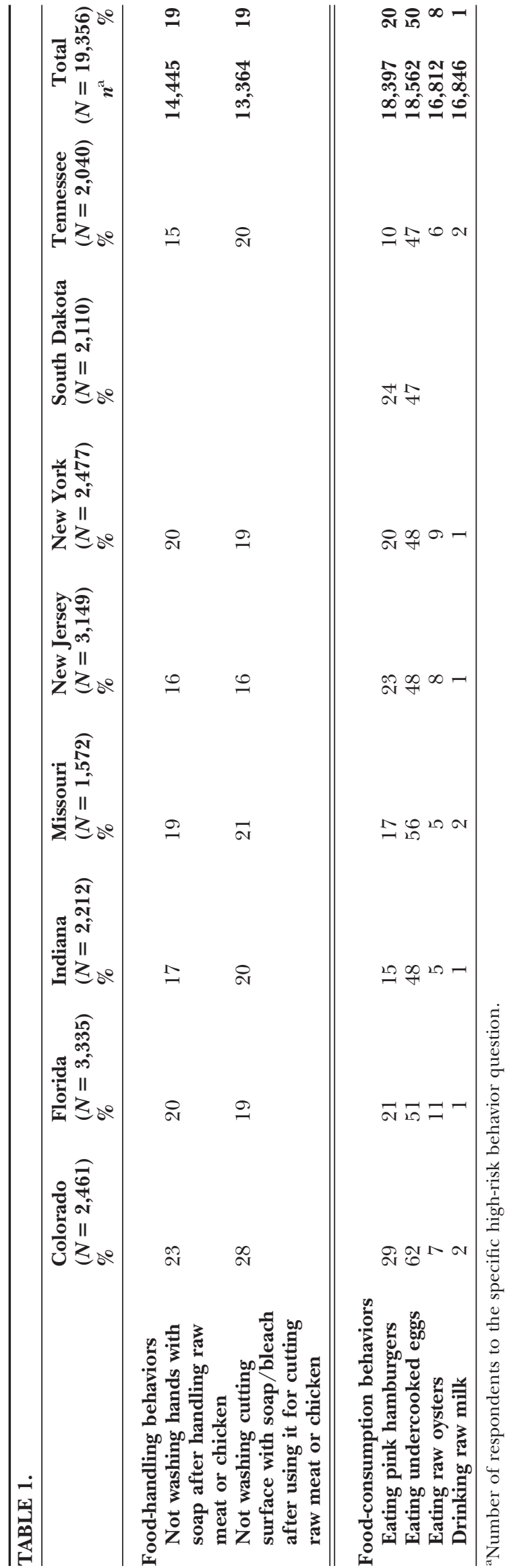

Am J Prev Med 1999;16(3) 


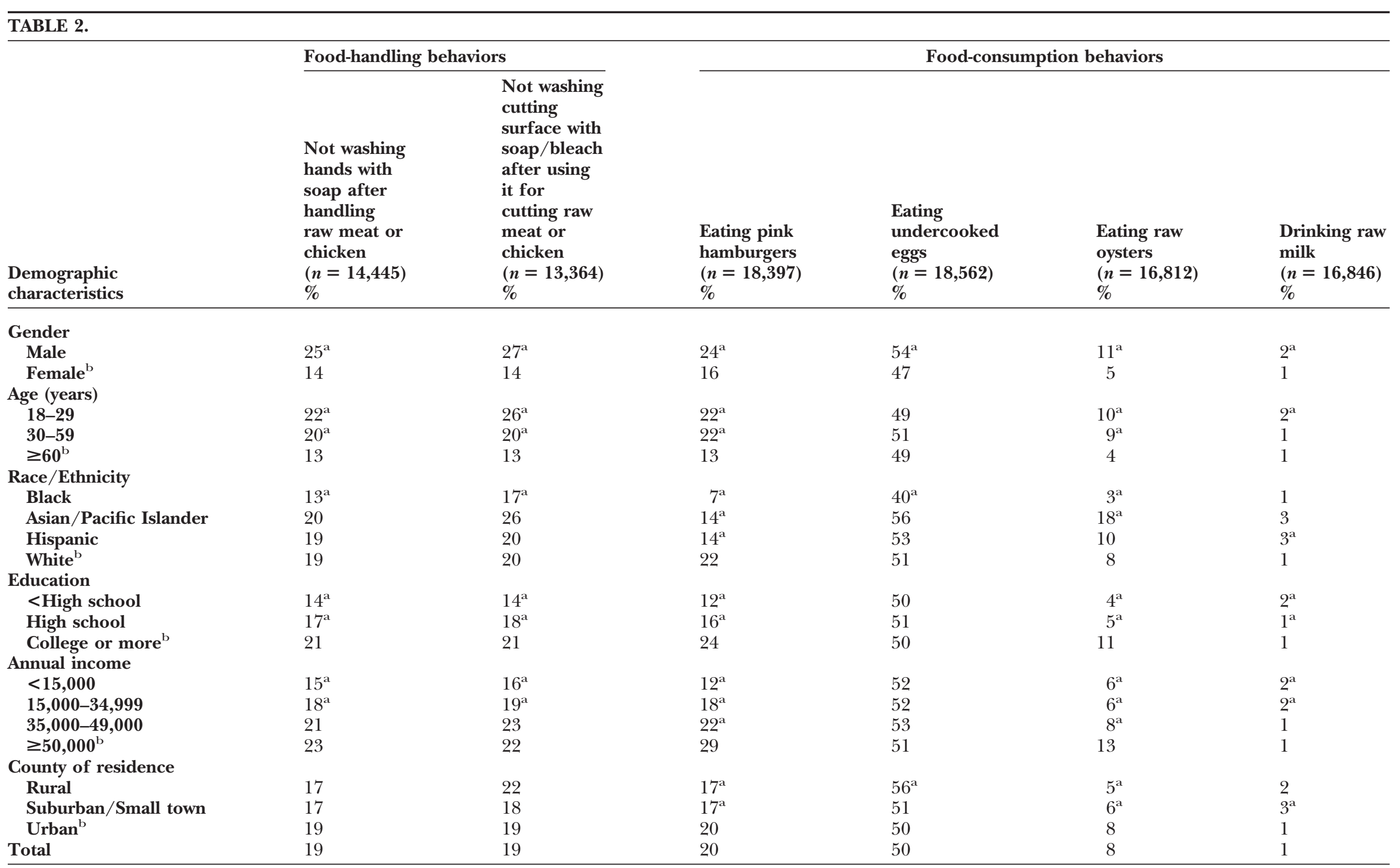

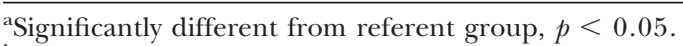

${ }^{b}$ Referent group. 
washing cutting boards with soap or bleach after cutting raw meat or chicken. The prevalence of this behavior varied from $16 \%$ in New Jersey to $28 \%$ in Colorado and was $20 \%$ in Tennessee, where data were available for approximately one half of respondents. Age- and gender-specific prevalences were similar to those for not washing hands after handling raw meat or chicken. The prevalence of the risky behavior was higher for men $(27 \%)$ than women $(14 \%)$, declined with increasing age, and increased with increasing education and increasing income.

Overall, $20 \%$ of respondents reported eating pink hamburgers during the previous 12 months. The prevalence of this risky behavior varied from $10 \%$ in Tennessee to $29 \%$ in Colorado. The prevalence was significantly higher for men (24\%) than women $(16 \%)$ and decreased with increasing age. In addition, the prevalence of the risky behavior was higher for whites $(22 \%)$ than blacks $(7 \%)$, increased with increasing education and increasing income, and was more common among residents of urban counties (20\%) than residents of rural counties $(17 \%)$.

Most respondents (50\%) reported eating undercooked eggs during the previous 12 months. Prevalences varied from $47 \%$ in South Dakota and Tennessee to $62 \%$ in Colorado. Eating undercooked eggs was more common among men (54\%) than women (47\%). Consumption of undercooked eggs was the only behavior we studied with no significant difference in agespecific prevalences; however, among consumers of undercooked eggs, $56 \%$ of persons $\geq 60$ years old reported eating undercooked eggs more than 4 times a month (median frequency), compared with $48 \%$ of adults $<60$ years old $(P<0.01)$. Eating undercooked eggs was significantly more common among whites $(51 \%)$ than blacks (40\%). In addition, this was the only behavior for which prevalences did not vary by income or education. The risky behavior was more prevalent among residents of rural counties $(56 \%)$ than residents of urban counties $(50 \%)$.

Few respondents $(8 \%)$ reported eating raw oysters during the previous 12 months. Prevalences varied from $5 \%$ in Indiana and Missouri to $11 \%$ in Florida. Consumption of raw oysters was more commonly reported by men $(11 \%)$ than women $(5 \%)$. Prevalence of this behavior decreased with increasing age. The behavior was more common among Asians/Pacific Islanders (18\%), compared with whites $(8 \%)$ and blacks $(3 \%)$, increased with increasing education and increasing income, and was more common among residents of urban counties $(8 \%)$ than residents of rural counties $(5 \%)$.

Drinking unpasteurized milk was reported by $1 \%$ of respondents and was the least common risky behavior. Prevalences varied from $1 \%$ in Florida, Indiana, New Jersey, and New York to $2 \%$ in Colorado and Missouri. Men $(2 \%)$ were significantly more likely than women
(1\%) to report drinking unpasteurized milk. Prevalence decreased with age. Drinking unpasteurized milk was more common among Asians/Pacific Islanders and Hispanics (3\%) than whites and blacks (1\%). Unlike other risky behaviors that we studied, prevalence decreased with increasing income and education. Drinking unpasteurized milk was more common among residents of suburban/small town counties $(3 \%)$ than among residents of urban counties (1\%).

\section{Discussion}

Risky food-handling and food-consumption behaviors were common among survey respondents. Men and young adults were most likely to report risky behaviors. Our findings suggest that many consumers could benefit from food-safety education. ${ }^{9}$

As in a nationwide survey conducted by the FDA, ${ }^{6}$ the prevalence of some risky behaviors (i.e., not washing hands and cutting boards after handling raw meat and poultry, eating pink hamburgers, and eating raw oysters) increased with increasing socioeconomic status. There are several possible explanations for this unexpected result. One plausible explanation is that safe food-handling and food-consumption practices are developed through food-preparation experience in the kitchen ${ }^{15}$ to a greater extent than from intellectual awareness of foodborne disease hazards. ${ }^{16}$ To the extent that persons in high socioeconomic strata do not prepare meals as often as persons in other strata, they may have a higher prevalence of risky food-handling and food-consumption behaviors. If food-preparation experience is an important determinant of safe foodhandling, it may have implications for the design of effective food safety educational efforts.

Similar to the FDA's 1993 survey, one in five respondents did not routinely wash hands or cutting boards after contact with raw meat or chicken. ${ }^{6}$ These practices facilitate the cross-contamination of foods by Campylobacter and Salmonella. The high prevalence of these behaviors among young adults, especially men, corresponds with their high rate of Campylobacter jejuni infection. ${ }^{17}$

Despite publicity about the risk of Escherichia coli O157:H7 infection associated with eating pink hamburgers, ${ }^{18}$ one in five respondents ate pink hamburgers during the previous year. While this is lower than the prevalence reported in a 1993 survey (29\%), the behavior was still common among men, persons of high socioeconomic status, urban residents, and whites. The low prevalence of pink-hamburger consumption among blacks may, in part, explain the lower rate of diarrhea-associated hemolytic uremic syndrome in blacks compared to whites. ${ }^{19}$ Consumer education and surveillance activities related to this behavior should continue.

As in a 1993 survey, ${ }^{6}$ the most commonly reported 
risky behavior in this survey was eating undercooked eggs, reported by approximately one half of respondents. The behavior was common in all demographic groups. Despite extensive data indicating that undercooked eggs are a major source of Salmonella serotype enteritidis, ${ }^{20-22}$ the incidence of Salmonella enteritidis infections continues to increase in the United States. The frequent consumption of undercooked eggs by older adults is of particular concern because the rate of invasive salmonellosis is particularly high in this age group. ${ }^{23}$

Raw oyster consumption (reported by $8 \%$ of respondents) is a risk factor for Vibrio vulnificus primary septicemia, a rare but often fatal syndrome, affecting primarily patients with liver disease. ${ }^{24}$ Raw oyster consumption is also a risk factor for Vibrio gastroenteritis ${ }^{24}$ and viral gastroenteritis, ${ }^{25}$ syndromes not associated with underlying disease. As in other studies, most raw oyster consumers were male, young or middle-aged adults, and persons of high socioeconomic status. ${ }^{25}$ As in a California study, Asians/Pacific Islanders and Hispanics were more likely to eat raw oysters than other racial/ethnic groups. ${ }^{26}$ Several factors may impede consumer education campaigns about the risk of raw oyster consumption including the association between this behavior and other risk-taking behaviors, particularly related to alcohol. ${ }^{25,26}$ In addition, point-of-sale warning signs about the hazards of raw oyster consumption may not reach vulnerable populations. ${ }^{27}$ It may be useful to institute processing steps to reduce pathogen counts in molluscan shellfish (e.g., cold shock, heat shock, irradiation).$^{28}$ The need for a pathogen kill step is particularly important for oysters and clams marketed "raw on the half shell" in bars and restaurants.

Less than $2 \%$ of respondents drank unpasteurized milk during the previous 12 months. This behavior is associated with Campylobacter, Salmonella, and E. coli O157:H7 infections. ${ }^{29}$ Similar to findings in a California study, the behavior was most prevalent among young adults, Hispanics, and persons of low socioeconomic status. ${ }^{30}$ The association with non-urban residence suggests that the dairy farm may be an immediate source of unpasteurized milk. This is compatible with findings from a study of campylobacteriosis in Iowa. ${ }^{31}$ Regulations restricting farm sales ${ }^{29}$ and restricting the use of raw milk to compensate employees may have merit.

Limitations of our study included the use of selfreported behaviors, which may or may not reflect actual behaviors and/or food preferences. Nonetheless, the high prevalence of self-reported risky behaviors emphasizes the potential value of food safety educational efforts. Because risky behaviors are common, consumer education is only one component of a broad foodborne disease prevention strategy that includes sanitary food production on the farm and the control of hazards in processing, distribution, and at retail. ${ }^{32}$
The authors thank Georgette Boeselager, Mark Gildemaster, Patricia Griffin, Kathy Horvath, Jeanette Jackson-Thompson, Marilyn Leff, David McQueen, Doris McTague, Tom Melnik, Theophile Murayi, Morrie Potter, David Ridings, David Swerdlow, and Michael Waller for their contributions.

\section{References}

1. Busby JC, Roberts T, Lin C-T J, MacDonald JM. Bacterial Foodborne Diseases: Medical costs and productivity losses. Food and Consumer Economics Division, Economic Research Service, U.S. Department of Agriculture Report No. 741.

2. Altekruse SF, Cohen ML, Swerdlow DL. Emerging foodborne diseases. Emerging Infectious Diseases 1997; 3:285-93.

3. A report to the President, Food safety from farm to table: A National Food-Safety Initiative, 1997. United States Department of Agriculture/ Department of Health and Human Services. Washington, D.C. April 1997.

4. Partnership for food safety education. 1997. Fighting the problem of foodborne illness. World wide web site. "Fight BAC" web site: www.fightbac.org. Accessed October 23, 1998.

5. Williamson DM, Gravani RB, Lawless HT. Correlating food safety knowledge with home food-preparation practices. Food Technology 1992; 46: 94-100.

6. Klontz KC, Timbo B, Fein S, Levy A. Prevalence of selected food consumption and preparation behaviors associated with foodborne disease. J Food Protection 1995; 58:927-30.

7. Raab CA, Woodbury MJ. Changing risk perceptions and food handling practices of Oregon household food preparers. J Consumer Studies Home Econ 1997; 21:117-30

8. Albrecht JA. Food safety knowledge and practices of consumers in the U.S.A. J Consumer Studies Home Econ 1995; 19:119-34.

9. Goodman RA, Remington PL, Howard RJ. Communicating information for action. MMWR 1992; 41 (suppl):143-7.

10. Gentry EM, Kalsbeck WD, Hogelin GC, et al. The behavioral risk factor surveys. Part II. Design, methods, and estimates from combined state data. Am J Prev Med 1985; 1:9-14.

11. Cook PR. 1989 ERS typology codes. Washington, D.C.: United States Department of Agriculture, Economic Research Service, 1995.

12. SAS/STAT software: changes and enhancements through release 6.12. Cary, North Carolina: SAS Institute, Inc, 1997.

13. Shah BV, Barnwell BG, Bieler GS. SUDAAN User's Manual, Release 7.0. Research Triangle Park, North Carolina: Research Triangle Institute, 1996.

14. Behavioral Risk Factor Surveillance System. BRFSS Quality Control Report. Atlanta, Georgia: Centers for Disease Control and Prevention, National Center for Health Promotion and Disease Prevention, 1996

15. Altekruse SF. Effectiveness of consumer labels for the safety of foods of animal origin. J Am Vet Med Assoc 1996; 209:2056. [Abstract]

16. Altekruse SF, Street D, Fein S, Levy A. Consumer knowledge of foodborne microbial hazards and food-handling practices. J Food Protect 1996; 59:287-94

17. Tauxe RV, Pegues DA, Hargrett-Bean N. Campylobacter infections: the emerging national pattern. Am J Public Health 1987; 9:1219-21.

18. Bell BP, Goldoft M, Griffin PM, et al. A multi-state outbreak of Escherichia coli $\mathrm{O} 157$ :H7-associated bloody diarrhea and hemolytic uremic syndrome from hamburgers. the Washington experience. JAMA 1994; 272:1349-53.

19. Jernigan SM, Waldo FB. Racial incidence of hemolytic uremic syndrome. Pediatr Nephrol 1994; 8:545-7.

20. Hedberg CW, David MJ, White KE, MacDonald KL, Osterholm MT. Role of egg consumption in sporadic Salmonella enteritidis and Salmonella typhimurium infections in Minnesota. J Infect Dis 1993; 167:107-11.

21. Passaro DJ, Reporter R, Mascola L, et al. Epidemic illness caused by Salmonella enteritidis infection in Los Angeles County: the predominance of phage type 4. Western J Med 1996; 165:126-30.

22. Mishu B, Koehler J, Lee LA, et al. Outbreaks of Salmonella enteritidis infections in the United States, 1985-1991. J Infect Dis 1994; 169:547-52.

23. Blaser MJ, Feldman RA. From the Centers for Disease Control: Salmonella bacteremia-reports to the Centers for Disease Control, 1968-1979. J Infect Dis 1981; 143:743-6.

24. Hlady WG, Klontz KC. The epidemiology of Vibrio infections in Florida, 1981-1993. J Infect Dis 1996; 173:1176-83. 
25. Klontz KC, Desenclos JC, Wolfe LE, Hoecherl SA, Roberts C, Gunn RA. The raw oyster consumer-a risk taker?: use of the Behavioral Risk Factor Surveillance System. Epidemiology 1991; 2:437-40.

26. Timbo B, Altekruse SF, Headrick M, Klontz KC. Raw shellfish consumption in California: the 1992 California Behavioral Risk Factor Survey. Am J Prev Med 1995; 11:214-6.

27. Mouzin E, Mascola L, Tormey MP, Dassey DE. Prevention of Vibrio vulnificus infections: assessment of regulatory educational strategies. JAMA 1997; 278:576-8.

28. Oliver JD, Kaper JB. Vibrio species. In: Food Microbiology: fundamentals and frontiers. Doyle MP, Beuchat LR, Montville TJ, eds. Washington, DC: American Society for Microbiology, 1997:229-64.
29. Headrick ML, Korangy S, Bean NH, et al. Epidemiology of raw milkassociated foodborne disease outbreaks in the United States, 1973-1992. Am J Public Health 1998; 88:1219.

30. Headrick ML, Timbo B, Klontz KC, Werner SB. Profile of raw milk consumers in California. Public Health Rep 1997; 112:418-22

31. Schmid GP, Schaefer RE, Plikaytis BD, et al. A one-year study of endemic campylobacteriosis in a midwestern city: Association with consumption of raw milk. J Infect Dis 1987; 156:218-22.

32. Food and drug safety. In: Healthy People 2000. National health promotion and disease prevention objectives-full report with commentary. Washington, D.C.: U.S. Department of Health and Human Services, 1991; 342-8. DHHS publication no. (PHS) 91-50212. 